\title{
THE TREATMENT OF EPIPHORA WITH BALLOON DACRYOCYSTOPLASTY
}

\author{
R. ROBINSON ${ }^{1}$, N. TURNER ${ }^{2}$, P. BRETTLE ${ }^{3}$, P. B. CHELL ${ }^{1}$, S. V. CHAVDA ${ }^{1,3}$ and P. I. MURRAY ${ }^{1}$ \\ Birmingham
}

\begin{abstract}
SUMMARY
We examine a new treatment for epiphora: balloon dilatation of the nasolacrimal duct, or dacryocystoplasty. The procedure, which was performed as an out-patient procedure under local anaesthetic, was carried out on 20 patients with epiphora, 17 due to anatomical and 3 due to functional obstruction. There was an overall success rate of $60 \%$, with complete relief of symptoms in 4 patients and a symptomatic improvement in another 8 (follow-up 2-9 months, mean 4.2 months). All patients tolerated the procedure well with minimal per-operative and postoperative discomfort. A higher success rate was seen in those patients with either a low anatomical or a functional obstruction. The procedure can be easily repeated and, if unsuccessful, still allows a dacryocystorhinostomy to be performed at a later date.
\end{abstract}

Epiphora is a common out-patient referral and although not sight threatening can be considerably annoying to many patients. Dacryocystorhinostomy (DCR) is the standard treatment for epiphora resulting from obstruction of the nasolacrimal sac or nasolacrimal duct (NLD). ${ }^{1}$ This procedure is usually associated with a high success rate, ${ }^{2,3}$ but is generally performed on an in-patient basis under a hypotensive general anaesthetic, ${ }^{4}$ which may carry a higher risk of morbidity in an elderly population. Although DCR has been carried out as out-patient surgery, ${ }^{5}$ patients may require readmission, mainly for epistaxis.

Other forms of surgery for lacrimal drainage obstruction include silicone intubation, which may be performed on its own or in combination with DCR. Complications have also been reported with this technique. ${ }^{6,7}$ Lacrimal probing in adults has also been tried with varying degrees of success. ${ }^{8,9}$

From: ${ }^{1}$ Birningham and Midland Eye Hospital, Birmingham; ${ }^{2}$ Department of ENT, Dudley Road Hospital, Birmingham; ${ }^{3}$ Department of Radiology, Dudley Road Hospital, Birmingham, UK.

Correspondence to: Mr Philip I. Murray, Academic Unit of Ophthalmology, Birmingham and Midland Eye Hospital, Church Street, Birmingham B3 2NS, UK.
Recently, a new treatment for NLD obstruction has been described $^{10,11}$ which involves balloon dilatation of the obstructed duct using an angioplasty catheter, and has been termed balloon dacryocystoplasty (DCP). As this procedure can be performed as an out-patient procedure under local anaesthetic with few complications, we decided to undertake a prospective study of the technique in patients with epiphora.

\section{PATIENTS AND METHODS}

Patients attending the Birmingham and Midland Eye Hospital with moderate to severe epiphora of greater than 6 months' duration were entered into the study. Subjective epiphora was graded according to symptoms as shown in Table I. In each case the nasolacrimal system was syringed with normal saline. All patients demonstrated a resistance to the flow of saline, with regurgitation through the opposite punctum. To determine the site of obstruction, all patients had an erect pre-operative dacryocystogram (DCG) using lopiodol, an oil-based contrast medium. Where there was a definite stop, with no passage of contrast into the nasopharynx, the obstruction was classified as anatomical and the site of blockage was noted. In those cases where there was a free flow of contrast into the nasopharynx the obstruction was classified as functional. Patients with previous lacrimal surgery were excluded from the study. Ethics committee approval was granted and informed consent was obtained from each patient. In total, 20 patients ( 6 male, 14 female) were entered into the study including 2 patients with Wegener's granulomatosis. Ages ranged from 17 to 78 years (mean 51.8 years). The pre-operative DCG revealed anatomical obstruction

Table I. Subjective grading system for epiphora

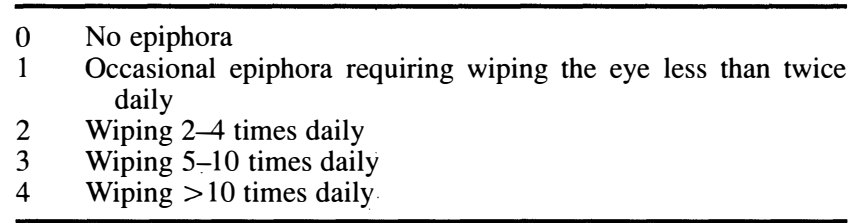




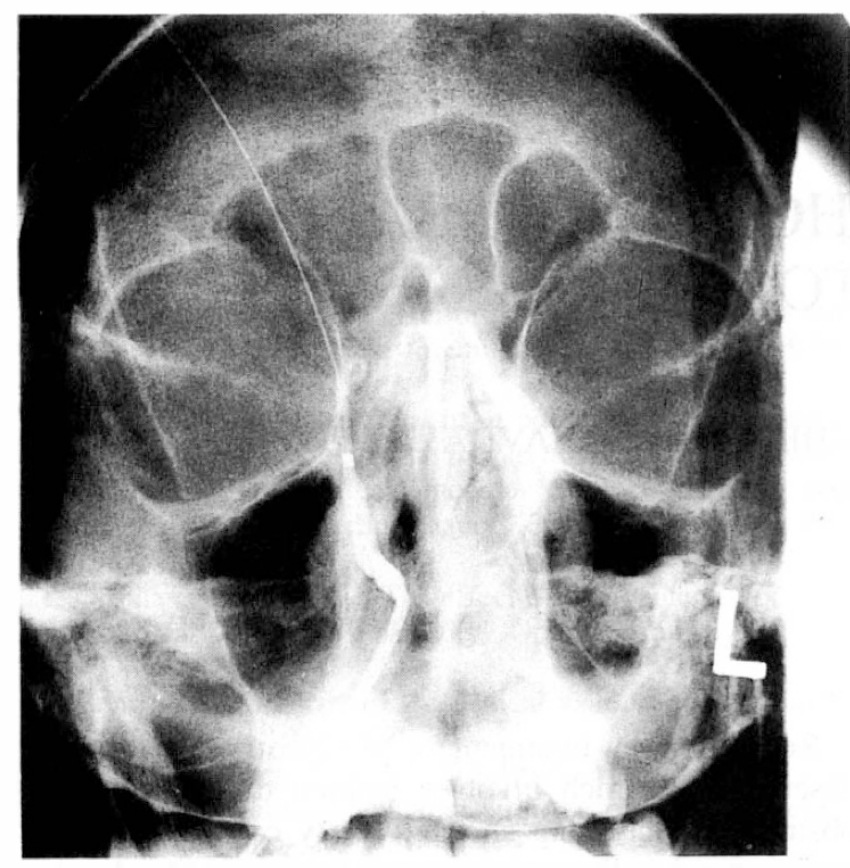

Fig. 1. Catheter in situ with balloon inflated.

in 17 (14 NLD, 3 mucocoeles) and a functional obstruction in 3 patients.

The procedure was performed under local anaesthesia as an out-patient procedure in the Department of Radiology, Dudley Road Hospital, Birmingham. Sedation was induced by $2-4 \mathrm{mg}$ of intravenous medazolam. Anaesthesia and vasoconstriction were achieved in the nose with $5 \%$ cocaine spray and packing. The inferior conjunctival fornix was instilled with benoxinate $0.4 \%$ and lignocaine $2 \%$ was infiltrated locally over the nasolacrimal sac. An infratrochlear block was given by injecting $2 \mathrm{ml}$ of lignocaine $2 \%$ medial to the caruncle at a depth of approximately $1.5 \mathrm{~cm}$. The lacrimal system was probed and syringed with a 21 gauge curved lacrimal cannula and passage into the nasal cavity was identified by free flow of saline which the patient tasted. A 0.018 inch guide wire was passed through the lacrimal cannula, located in the nose and brought forward through the nasal aperture. A 5 French tibial balloon angioplasty catheter, with a $4 \mathrm{~mm} \times 4 \mathrm{~cm}$ balloon located $15 \mathrm{~mm}$ from the tip, was then advanced, in a retrograde manner, along the wire into the NLD. Under X-ray control, the tip of the catheter was then passed into the lacrimal sac, and the balloon inflated with iopamidol contrast medium, dilating the distal half of the NLD (Fig. 1). The inflated balloon was left in situ for 3-4 minutes, then deflated and removed.

Post-operatively, topical antibiotic and steroid drops were administered for approximately 4 weeks in reducing

Table II. Pre- and post-operative grades of epiphora

\begin{tabular}{lcc}
\hline Grade & Pre-operative & Post-operative \\
\hline 0 & 0 & 4 \\
1 & 0 & 6 \\
2 & 3 & 2 \\
3 & 7 & 4 \\
4 & 10 & 4 \\
\hline
\end{tabular}

dosage. Patients were initially reviewed at 2 weeks and their overall tolerance of the procedure and any postoperative problems were noted. The post-operative degree of epiphora was documented. Patients were reviewed again at 3 months when a post-operative DCG was performed to determine patency of the system. Where patients did not have relief of symptoms at 2 weeks the procedure was repeated. Patients remaining symptom-free after 6 months were regarded as being cured.

\section{RESULTS}

In total, 27 procedures were performed, with 14 patients having 1 procedure, 5 having 2 procedures, and 1 having 3 procedures. Full relief of symptoms was seen in 4 patients, symptomatic improvement in 8 , and in another 8 symptoms remained unchanged (follow up 2-9 months, mean 4.2 months). In no patient was a worsening of symptoms seen. Table II shows the pre- and post-operative grading of epiphora.

During the procedure, hypertrophic inferior turbinates were noted in 9 patients. We were unable to pass the probe in 3 patients, including 1 patient with Wegener's granulomatosis and 2 with blind passages. No improvement in symptoms was seen in any of these 3 cases. In 2 patients the guide wire entered the nose submucosally. The balloon could not be passed in 3 cases, which included the other patient with Wegener's granulomatosis, 1 patient with a deviated nasal septum and very narrow access, and the first patient in the series. Despite this, 2 of the patients had improvement in symptoms.

All but one of the patients were pleased with the procedure, and experienced minimal per-operative and postoperative discomfort. The patient who was dissatisfied was drowsy for some hours afterwards, and as this was an out-patient procedure she felt this was not appropriate. Discomfort on insertion of the balloon was experienced in 2 patients, but additional anaesthesia was not required. Only 1 patient complained of pain requiring mild analgesia for approximately 1 week after surgery, and this was one of the patients in whom we had difficulty in passing the probe. Acute dacryocystitis developed 1 month after the procedure in 1 patient; this may have been unrelated to the surgery itself but due to persistence of a small mucocoele.

Table III shows the degree of success of the procedure in relation to the site of obstruction.

\section{DISCUSSION}

In this group of patients we have shown that DCP can be performed as an alternative to DCR. Other simple options in the treatment of NLD obstruction such as probing, although successful in children, ${ }^{12,13}$ have had varied results

Table III. Success vs site of obstruction

\begin{tabular}{ll}
\hline Functional & $2 / 3(66 \%)$ \\
Lower NLD & $7 / 8(87.5 \%)$ \\
Upper NLD & $3 / 8(37.5 \%)$ \\
\hline
\end{tabular}

NLD, nasolacrimal duct. 
in adults. Hanafee and Dayton ${ }^{8}$ had limited success in dilating the NLD with probes, but Bell ${ }^{9}$ performed probing on 26 patients with improvement in symptoms in $52 \%$ of cases. Unfortunately, the latter study failed to clarify the initial or residual degree of epiphora in these patients.

The use of stents in lacrimal surgery is not new. Many different materials including gauzes, rubber catheters, silicone tubing, absorbable gelatin-thrombin sponges and Y-shaped catheters have been used to keep open the anastomosis between the lacrimal sac and the nasal cavity. A no. 8 paediatric Foley catheter was used by Vila-Coro et $a l .{ }^{14}$, but Becker and Berry ${ }^{15}$ first described the use of an angioplasty catheter in this situation. They used the catheter successfully to dilate 4 cases of failed DCR due to stenosis of the nasal ostium.

In a recent study, Munk et al..$^{10}$ described the use of a tibial angioplasty catheter to dilate the NLD, with an improvement of symptoms in 13/16 patients. Their series differs from ours in that all but 2 of their cases were patent on pre-operative DCG, indicating a functional obstruction only. In their technique they described the passage of a guide wire without initial probing. In our study we found it impossible to pass the guide wire alone against an anatomical obstruction, with the wire coiling in the nasolacrimal sac. Song et al. ${ }^{11}$ experienced similar problems with passage of the guide wire. To overcome the difficulty they used ball-tipped guide wires. We probed the system first with a nasolacrimal cannula and then passed the guide wire down through the cannula.

Song et al. ${ }^{11}$ had an initial success rate of 22/39 $(56 \%)$, but a 2 -month recurrence rate of $45 \%$. In our series, all patients who were free of symptoms or improved at 2 weeks after the procedure remained so at 3 months. Song et al. ${ }^{16}$ treated patients with recurrent obstruction by using metallic stents to maintain patency of the system.

In our series, the pre- and post-operative DCGs were retrospectively analysed to determine possible causes of failure. In 3 patients with functional obstruction, improvement was seen in 2; we were unable to locate the guide wire in the nose in 1 case. A middle to lower anatomical obstruction was noted in 8 patients, and 7 of these showed an improvement in symptoms. Another 8 cases had an upper NLD obstruction, but only 3 cases in this group improved. All cases of middle or lower NLD obstruction or functional obstruction were patent on the post-operative DCG. Of those with upper NLD obstruction, 5/7 were not patent (1 post-operative DCG was not performed). In retrospect, we now feel that the procedure may be limited in its present form to patients with functional obstruction or patients with middle or low NLD obstruction. This also explains the better results achieved by Munk et al. ${ }^{10}$ In our cases of upper NLD obstruction the tip of the catheter is in the sac but the balloon is not reaching the site of obstruction and so does not benefit the patient. We are at present negotiating with the manufacturers to design a catheter with the balloon located nearer the tip of the catheter to improve our results further.
The patients with Wegener's granulomatosis included in the study both had mucocoeles. They also had severe respiratory symptoms and therefore were unfit for general anaesthesia and DCR. Both had active disease and were receiving cytotoxic agents. In 1 case we were unable to pass the probe successfully and this patient had no relief of symptoms. In the second case we were able to pass the guide wire but were unable to use the balloon. This patient had relief of symptoms for 3 months. The treatment of NLD obstruction in patients with Wegener's granulomatosis is controversial. DCR has been successful in some patients with long-standing quiescent disease but is associated with nasal-cutaneous fistula formation in others. ${ }^{17}$ Although this present technique is difficult in these patients, it offers a less invasive alternative to DCR. It is difficult to assess success on the basis of only 2 cases, but as the procedure is easily repeatable, even relief of symptoms for a short period of time can make life more pleasant for the patient. Relief of symptoms may last longer in patients with quiescent disease.

In the present age of minimally invasive microsurgery, with early rehabilitation of the patient, there is a move away from traditional DCR. Recent reports ${ }^{18,19}$ advocate the success of endoscopic DCR using a nasal approach with or without the use of lasers. Although this technique allows early rehabilitation, it requires expensive equipment not readily available in most departments and is associated with a steep learning curve. The advantages of DCP are that it is easy to perform, is a day case procedure performed under local anaesthesia and is of low cost. It also preserves the normal nasolacrimal anatomy, can be repeated easily and, if unsuccessful, still allows a DCR to be performed. Our results indicate that DCP might have a role to play in the management of patients with epiphora.

Key words: Dacryocystoplasty, Epiphora, Nasolacrimal duct obstruction, Therapy.

\section{REFERENCES}

1. Tanenbaum M, McCord CD. The lacrimal drainage system. In: Tasman W, Jaeger EA, editors. Duane's clinical ophthalmology. Vol. 4. Philadelphia: Lippincott 1991:27-30.

2. Rosen N, Sharir M, Moverman D, Rosner M. Dacryocystorhinostomy with silicone tubes: evaluation of 253 cases. Ophthalmic Surg 1989;20:115-9.

3. Zolli CL, Shannon GM. Dacryocystorhinostomy: a review of 119 cases. Ophthalmic Surg 1982;13:905-10.

4. Welham RAN, Wulc AE. Management of unsuccessful lacrimal surgery. Br J Ophthalmol 1987;71:152-7.

5. Dresner SC, Klussman KG, Meyer DR, Linberg JV. Outpatient dacryocystorhinostomy. Ophthalmic Surg 1991; 22:222-4.

6. Anderson RL, Edwards JJ. Indications, complications and results with silicone stents. Ophthalmology 1979; $86: 1474-87$.

7. Veloudios A, Harvey JT, Philippon M. Long-term placement of silastic nasolacrimal tubes. Ophthalmic Surg 1991;22: 225-7.

8. Hanafee WN, Dayton GO. Dilating the nasolacrimal duct under radiological control. Radiology 1978;127:813-5.

9. Bell TAG. An investigation into the efficacy of probing the nasolacrimal duct as a treatment for epiphora in adults. Trans Ophthalmol Soc UK 1986;105:494-7. 
10. Munk L, Lin DTC, Morris DC. Epiphora: treatment by means of dacryocystoplasty with balloon dilation of the nasolacrimal drainage apparatus. Radiology 1990;177: 687-90.

11. Song HY, Ahn HS, Park CK, Kwon SH, Kim CS, Choi KC. Complete obstruction of the nasolacrimal system. I. Treatment with balloon dilation. Radiology 1993;186:367-71.

12. Kraft SP, Crawford JS. Silicone tube intubation for disorders of the lacrimal system in children. Am J Ophthalmol 1982;94:290-9.

13. Robb RM. Probing and irrigation for congenital nasolacrimal duct obstruction. Arch Ophthalmol 1986;104:378-9.

14. Vila-Coro AA, Sevilla MAG, Guijo MRB, Vila-Coro AA. Inflatable catheter for dacryocystorhinostomy. Arch Ophthalmol 1988;106:692-4.
15. Becker BB, Berry FD. Balloon catheter dilatation in lacrimal surgery. Ophthalmic Surg 1989;20:193-8.

16. Song HY, Ahn HS, Park CK, Kwon SH, Kim CS, Choi KC. Complete obstruction of the nasolacrimal system. II. Treatment with expandable metallic stents. Radiology 1993; 186:372-6.

17. Hardwig PW, Bartley GB, Garrity JA. Surgical management of nasolacrimal duct obstruction in patients with Wegener's granulomatosis. Ophthalmology 1992;99:133-9.

18. Metson R. Endoscopic surgery for lacrimal obstruction. Otolaryngol Head Neck Surg 1991;104:473-9.

19. Gonnering RS, Lyon DB, Fisher JC. Endoscopic laserassisted lacrimal surgery. Am J Ophthalmol 1991;111: 152-7. 